\title{
Testing and Evaluation for the Atomic Clock in Different Location
}

\author{
Wei Li \\ National Time Service Center/ Key Lab of Time-frequency \\ Standard of the Chinese Academy of Sciences, \\ Xi'an, China \\ kim_weili@ntsc.ac.cn
}

\author{
Yongliang $\mathrm{Xu}$ \\ National Time Service Center/ Key Lab of Precision \\ Navigation and Timing Technology of the Chinese \\ Academy of Sciences, Xi'an, China \\ xuyl@ntsc.ac.cn
}

\author{
Shifeng Li \\ National Time Service Center/ Key Laboratory of \\ Precision Navigation and Timing Technology of \\ the Chinese Academy of Sciences, \\ Xi'an, China \\ lisf@ntsc.ac.cn
}

\begin{abstract}
The national standard time, UTC (NTSC) kept by NTSC takes part in the international time comparison for a long time, which has high stability and accuracy. It can test and evaluate the atomic clock in different location through the technology of remote time comparison. In the BPL system, time-frequency standard is generated by the cesium atomic clock whose performance has a direct impact on time service precision. In addition, according to the international telecommunications union (ITU) requirement, broadcasting clock is needed to keep to the UTC within 100ns. In this paper, the testing criteria, data collection, processing method, performance evaluation parameter and calculation model are studied. Relying on the high-performance time standard of NTSC and the GPS CV link, the BPL broadcasting clock testing and evaluation system is set up, the time difference of broadcasting clock and UTC (NTSC) is got, and the time difference of broadcasting clock and UTC is got through the international time comparison link of NTSC. According to the all kinds of data, the performance of broadcasting clock is evaluated, the results shows that the broadcasting clock stability reach to the $3.3 e-14$ a day, $1.7 e-14$ in 5 days, the time deviation to UTC is within 20ns.
\end{abstract}

Keywords- Test; performance evaluation; GPS CV

\section{INTRODUCTION}

Chinese coordinated universal time, UTC (NTSC) as a national standard time is kept by the national time service center, which takes part in international time comparison for a long time, has a very high degree of stability and accuracy.

The BPL long system built in our country is the groundbased high precision time services system using loran-c pulse signal first time. In BPL systems, time and frequency standard is produced by the cesium atomic clock, as the broadcasting clock, its performance has a direct impact on time service precision. In addition, as a important time service system, according to the requirement of ITU ${ }^{[1]}$ to time service system, the deviation of BPL broadcasting time to UTC should be within 100 nanoseconds.

In this article, the BPL broadcasting clock is tested and its performance is evaluated through comparison with UTC (NTSC) using the remote time comparison method.

\section{The BASIC CRITERIA For Testing AND Evaluation}

In the time and frequency measurement, the frequency standard or clock to be measured is called the device under test. The measurement is a process to compare the device under test and the standard (or reference), two elements must be paid attention to as follows:

\section{A. Reference selection}

The accuracy of the reference must be at least 3 times higher than the object measured; one order of magnitude is the best.

\section{B. Measurement method selection}

Some time comparison method is needed to measure the object under test, the error of the time comparison links must be less than the object being measured 3 times. There are two methods to meet that requirement.

(1) Choose high-precision time comparison method, to meet the requirements of testing and evaluation.

(2) Optimizing the measurement method, accumulating measurement data, and use mathematical method to process the measurement data to reduce various types of errors.

\section{Testing And Evaluation Methods of BPL BROADCASTING CLOCK}

\section{A. Basic principle}

According to the basic principles of testing and evaluation, the UTC (NTSC) is regarded as the reference, the BPL broadcasting clock as the object under test. The time difference between the CLOCK ( BPL) and UTC 
(NTSC) is obtained through GPS CV link, it is shown as Figure 1.

If there are two GPS CV receiver in the ground station A and $\mathrm{B}$ with precise coordinates. The two stations observe the same satellite at the same time. So we can get the follows:

$$
\begin{aligned}
\Delta t_{i A} & =t_{i}-t_{A} \\
\Delta t_{i B} & =t_{i}-t_{B}
\end{aligned}
$$

Formula (1) is the time difference of satellite $\mathrm{i}$ and $\mathrm{A}$, formula (2) is the time difference of satellite $i$ and $B$.

Using formula (1) - formula (2), the time difference of A and $\mathrm{B}$ is $t_{B A}$ :

$$
\Delta t_{i A}-\Delta t_{i B}=\left(t_{i}-t_{A}\right)-\left(t_{i}-t_{B}\right)=t_{B}-t_{A}=t_{B A}
$$

GPS CV can completely offset clock error, the most of the ephemeris error, can also partly offset the ionosphere delay and tropospheric delay error ${ }^{[4]}$, this is the main reason why the GPS CV has the high precision.

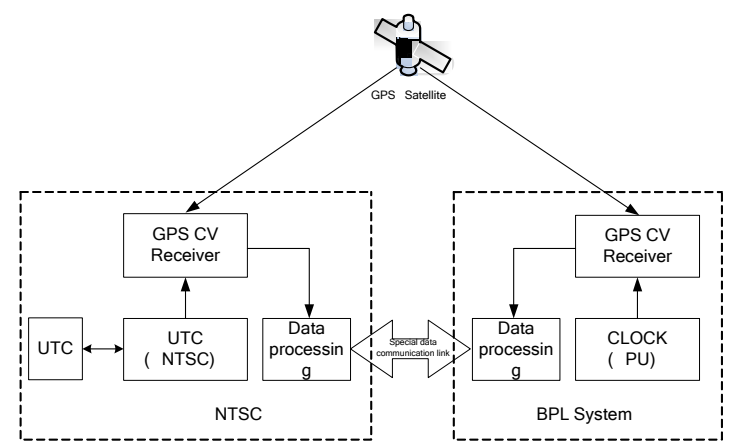

Figure 1. The principle diagram of broadcasting clock testing and evaluation

\section{B. GPS CV method}

1) GPS CV basic principle. GPS CV method was put forward by the American National Standards Institute in $1980^{[2]}[3]$, its basic principle is shown as figure 2 . The two ground station receive the same satellite signal at the same time, then the time comparison between two atomic clocks are fulfilled through data exchange.

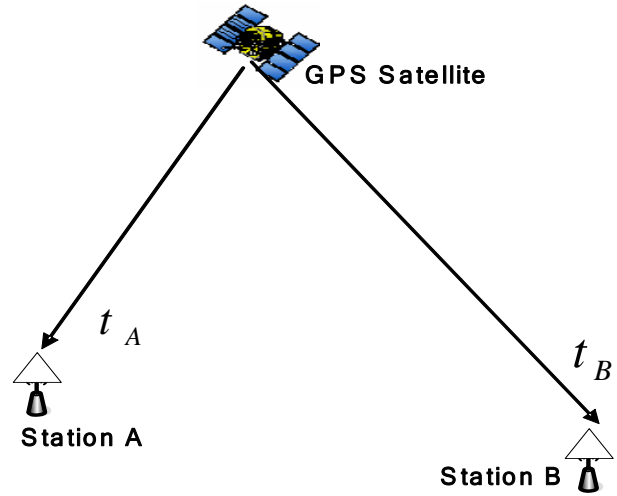

Figure 2. The principle diagram of GPS CV
2) The processing method of receiver internal observation data. The REFGPS in the file outputted by GPS receiver is the time difference of CLOCK (BPL) and satellite $i$ 、UTC (NTSC) and satellite $i$, which have been added the several modified items. The CLOCK (BPL) - UTC (NTSC) is calculated through the selection of the same GPS CV file according to the output files of two GPS CV receivers, including the PRN (satellite number), satellite elevation, CL (types), MJD, HHMMSS (start time tracking) and TRKL (trace length), etc.

3) The smoothing method of GPS CV data. Because of the measurement noise and various kinds of measurement errors (such as the ionosphere additional time delay, etc.), the real-time data can't reflect the quality level of atomic clocks itself. GPS CV needs to process the data more than one day using the method of statistical analysis after the exchange of observation data to get a better time comparison results. So the data processing method has become the key to raise the transmission precision. There are many smoothing filter methods, Vondrak smoothing method is the usual method used internationally.

Vondrak smoothing method is a method of curve fitting to eliminate the random error in the measurement data. This method can smooth the measurement data in a reasonable manner in the case of unknown form fitting function. Vondrak smoothing essentially has the function of unilateral filter in data processing, it can be used as a digital filter. This method is put forward by Vondrak, a astronomer of Czechoslovakia in $1969^{[5]}$.

Supposing there is measurement data $x_{i}(i=1,2,3 \ldots N)$, the fundamental of Vondrak smoothing method is:

$$
\begin{aligned}
& Q=F+\lambda^{2} s=\min \\
& F=\sum_{i=1}^{N} P_{i}\left(x_{i}^{\prime}-X_{i}\right)^{2} \\
& S=\sum_{i=1}^{N-3}\left(\Delta^{3} X_{i}^{\prime}\right)^{2}
\end{aligned}
$$

As the formula shown, $X_{i}$ is the unknown smoothed value, $P_{i}$ is the weight of the measured data.

$\mathrm{F}$ is Vondrak smoothing goodness of fit. S represents the sum of third-order differential square of smoothed value, if the third-order differential of a curve is very small, then obviously this curve is very smooth, so that $\mathrm{S}$ is the smoothness. $\lambda^{2}$ is a given positive coefficient, which adjust the relationship between the goodness of fit and the degree of smoothing. In the extreme case, when $\lambda^{2}$ tends to $\infty$, to make the formula (4) up to the minimum value, $S$ must tends to 0 , then a smooth quadratic parabola is got through the Vondrak smoothing. Conversely, if $\lambda^{2}$ tends to 0 , to make the formula (4) up to the minimum value, F must be close to zero, then a smooth is got.

It is shown from the Vondrak smoothing basic principles that this method is to seek a compromise curve, which is 
between the absolute curve approximation of the measurement data and absolute curve smoothness, the degree of compromise is controlled by $\lambda^{2}$. The formula (4) is a combination distribution, the idea of Vondrak smoothing method make the combination distribution to reach the minimum value to estimate a reasonable smooth curve.

\section{Index calculation model}

1) Accuracy and calculation model. Frequency accuracy refers to the consistency degree between the output frequency of device under test and the nominal frequency, it indicates the correctness of the oscillator output. The method to calculate the frequency accuracy by time difference comparison is called time difference method. The details to calculate are as follows:

$\widehat{x}(t)$ is the compared broadcasting clock time differences, after gross error elimination of the those data, least squares linear fitting is used, and the fitting method is:

If

$$
\bar{t}_{i}=\frac{1}{N} \sum_{i=1}^{N} t_{i}, \quad \bar{x}_{i}=\frac{1}{N} \sum_{i=1}^{N} x_{i}(\tau), \quad \mathrm{i}=1,2,3 \ldots \ldots . \mathrm{N}
$$

Draw a straight line through the point $\left(\bar{t}_{i}, \bar{x}_{i}\right)$, the equation is:

$$
x(t)=\bar{x}_{i}+k\left(t-\bar{t}_{i}\right)
$$

While

$$
k=\frac{\sum\left(x_{i}-\bar{x}_{i}\right)\left(t-\bar{t}_{i}\right)}{\sum\left(t-\bar{t}_{i}\right)^{2}}
$$

Taking zero result noted as $\widehat{x}(t)$ after fitting. The broadcasting clock time difference data is affected by the frequency drift rate, so the data for fitting should not be too long. Make $\tau=1$, one data $\hat{x}_{i}(t)$ one day is obtained by fitting, $\mathrm{i}=1,2,3 \ldots . \mathrm{N}$ (day).

Calculating frequency accuracy by comparing the time difference data, the formula is:

$$
\begin{gathered}
A=f(\text { offest })=\frac{-\Delta t}{T} \\
=\frac{\widehat{x}\left(t_{2}\right)-\widehat{x}\left(t_{1}\right)}{t_{2}-t_{1}}=\frac{\widehat{x}(t+\tau)-\widehat{x}(t)}{\tau}
\end{gathered}
$$

$\widehat{x}(t)$ is the time difference data after processing, $\tau$ is the sampling time. A number of frequency accuracy can be obtained by using $\tau$ as sampling interval to calculate. The average of these frequency accuracy is taken as the final evaluation result.

2) stability and calculation model. Stability characterizes the ability of the atomic clock to generate the same time and frequency within a certain time, and describes the random fluctuations of atomic clock output frequency. It does not indicate the time or frequency accuracy or not, only on whether the same value. Standard deviation of the data group is used to represent the stability in some occasions, but standard deviation can only used for stationary data. Because frequency offset contains time-varying noise, oscillator data are usually non-stationary. For stationary data, the number of measurements increases, the mean and variance converge to the same value; for non-stationary data, the mean and variance does not converge to a certain value. Conversely, when increasing the number of measurements, the average value will be slidably changed. For this reason, some nonclassical methods are used to represent time domain stability of time frequency signal. That statistical properties is called Allan variance, and it is the variance calculated by the time difference dada sampled for the second time.

$$
\sigma_{x}(\tau)=\frac{1}{\tau} \sqrt{\frac{1}{2(N-2)} \sum_{i=1}^{N-2}\left(\widehat{x}_{i+2}-2 \widehat{x}_{i+1}+\widehat{x}_{i}\right)^{2}}
$$

\section{RESUlts}

The time difference between UTC(NTSC) and CLOCK(BPL) from July 14, 2012 to September 1 is processed and the result is show as figure 3 .

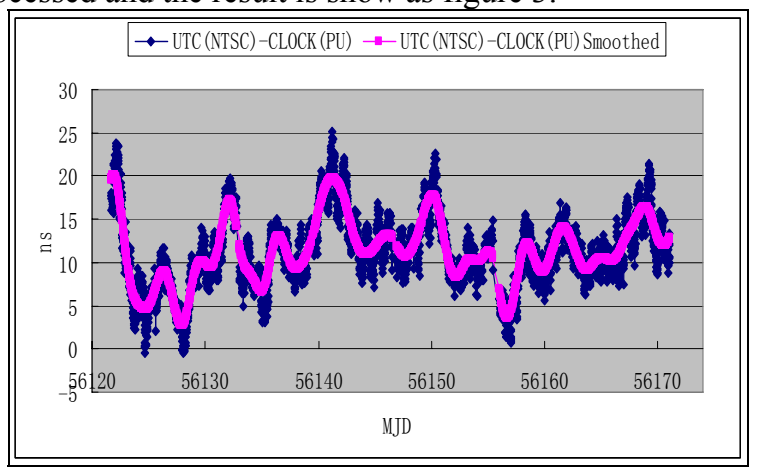

Figure 3. The time difference of UTC(NTSC) and CLOCK(BPL)

The calculating result of index is shown as figure 4 and table 1

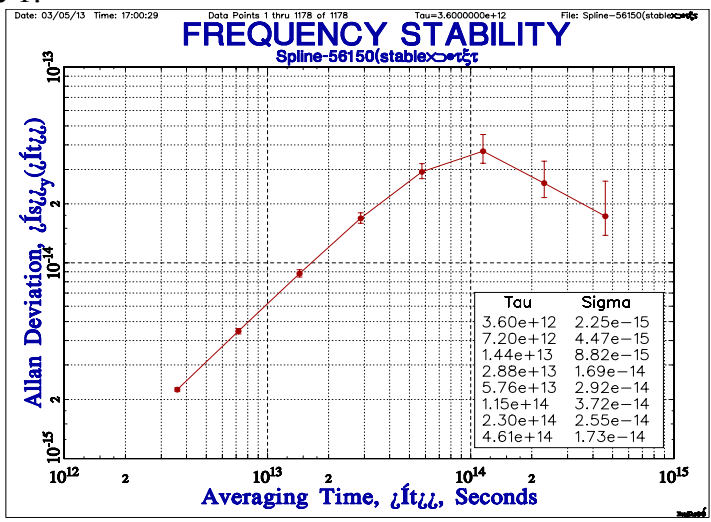

Figure 4. stability of clock(BPL)

Table 1. The accuracy and stability of clock(BPL)

\begin{tabular}{|c|c|c|}
\hline Time & $\begin{array}{c}\text { Frequency } \\
\text { accuracy }\end{array}$ & $\begin{array}{c}\text { Frequency } \\
\text { stability }\end{array}$ \\
\hline $\begin{array}{c}2012.7 .14-2012.9 .1 \\
\text { MJD (56122-56171) }\end{array}$ & $3.525 \mathrm{E}-14$ & $3.3 \mathrm{e}-14(1 \mathrm{~d})$ \\
\cline { 3 - 3 } & & $1.7 \mathrm{e}-14(5 \mathrm{~d})$ \\
\hline
\end{tabular}


The result of time difference between UTC and CLOCK(BPL) is shown as figure 5.

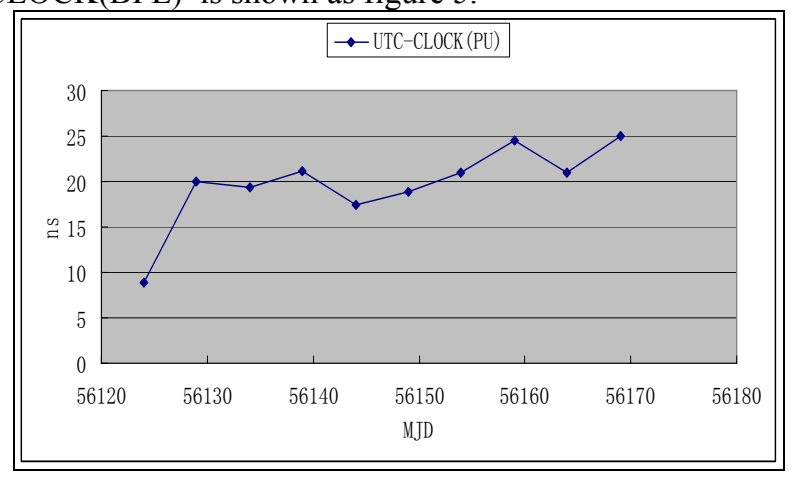

Figure 5. The time deviation of UTC and CLOCK(BPL)

\section{CONCLUSIONS}

In this experiment, the evaluation results show that the accuracy of BPL broadcasting clock can reach the magnitude of $3.525 \mathrm{E}-14$. The stability result evaluated by Allan Variance shows that the stability of BPL broadcasting clock reach $3.3 \mathrm{e}-14$ in one day and reaches the highest level of $1.7 \mathrm{e}-14$ in five days time interval.
BPL broadcasting clock keep within 20ns with UTC deviation, far more than the ITU requirement for time service system. Performance evaluation and assessment index contains the UTC (NTSC) and GPS CV link influence, so actual indicators should be superior to the evaluation results.

\section{ACKNOWLEDGMENT}

We thank Dr. Yuan haibo for the help on this article.

\section{REFERENCES}

[1] Radio communication Bureau. ITU handbook of selection and use of precise frequency and time system[M] . 1997.

[2] D. W. Allan and M. A. Weiss, "Accurate Time and Frequency Transfer During Common-View of a GPS Satellite," Proceedings of the 1980 Frequency Control Symposium, pp: 334-346, May 1980

[3] [Judah Levine. A review of time and frequency transfer methods. Metrologia 45 (2008), S162-S174.

[4] David W. Allan and Marc A.Weiss. Accurate time and frequency transfer during common-view of a GPS satellite. Proc. 34th Annual. Freq.Control Symposium. pp:334-346.

[5] Vondrak J. Problem of smoothing observational data [J]. Bull. Astron,Inst. Czech. 1969, 20(6): 349 